\title{
Review of: "Small heterodimer partner (SHP) aggravates ER stress in Parkinson's disease-linked LRRK2 mutant astrocyte by regulating XBP1 SUMOylation"
}

\author{
Peter Racay ${ }^{1}$ \\ 1 Comenius University in Bratislava
}

Potential competing interests: The author(s) declared that no potential competing interests exist.

The present manuscript describes a novel regulatory mechanism involving XBP1 in LRRK2-G2019S mutant astrocytes and highlights the importance of the SHP/PIAS1/XBP1 axis in some models of PD. The study seems to be well performed and provides some new information about pathophysiology of PD. There are some minor problems with presentation and interpretation of obtained results.

Minor comments:

Evaluation of Western blots is not clearly described. Authors should add some detail about WB evaluation. SUMO is $12 \mathrm{kDa}$ in mass. Modification of XBP1 related to SUMO can be seen on Fig. 3B but not on Fig 2C. Is there a particular reason?

Fig 4D CHOP expression looks to be depressed by inhibition of all three arms of UPR. This opens the questions about either specificity of inhibitors or performance of experiments.

Fig 4E results for WT cell treated with siSHP are not shown. Is there a particular reason? In addition, the extend of SHP silencing caused by siSHP is not neither shown nor described.

Doxycycline inhibits mitochondrial translation and thus induces integrated stress response that depends on AFT4 (Guo X, Aviles G, Liu Y, Tian R, Unger BA, Lin YT, Wiita AP, Xu K, Correia MA, Kampmann M. Mitochondrial stress is relayed to the cytosol by an OMA1-DELE1-HRI pathway. Nature. 2020 Mar;579(7799):427-432. doi: 10.1038/s41586-020-2078-2.). This can interfere with PERK arm of UPR. Authors should either consider this fact or comment this fact.

The next sentence is a bit confusing and should be modified that the information is clearer. One of the sensors, IRE1, activates the transcription factor XBP1 to induce genes that facilitate protein folding and removal of unfolded proteins by ER-associated degradation by ER-associated degradation (ERAD), including some that encode ER chaperones [14, 42, 45, 54].

The next sentence does not reflect reality. Another UPR sensor, PERK, drives apoptotic cell death through ATF4-dependent induction of the transcription factor CHOP, which induces expression of caspases [38]. In fact, CHOP does not affect expression of caspases. With respect to mitochondrial apoptosis, it positively regulates transcription of genes coding for pro-apoptotic proteins of Bcl2 family ( $L i$ J, Lee B, Lee AS 
Endoplasmic reticulum stress-induced apoptosis: multiple pathways and activation of p53-up-regulated modulator of apoptosis (PUMA) and NOXA by p53. J Biol Chem 2006, 281, 7260- 7270. Galehdar, Z. et al. Neuronal apoptosis induced by endoplasmic reticulum stress is regulated by ATF4-CHOP-mediated induction of the Bcl-2 homology 3-only member PUMA. J. Neurosci. 2010, 30, 16938-16948. Ghosh, A. P., Klocke, B. J., Ballestas, M. E. \& Roth, K. A. CHOP Potentially Co-Operates with FOXO3a in Neuronal Cells to Regulate PUMA and BIM Expression in Response to ER Stress. PLoS One 2012, 7, e39586) and negatively regulates transcription of genes coding for anti-apoptotic proteins of Bcl2 family (Gomez-Bougie P, Halliez M, Moreau P, Pellat-Deceunynck C, Amiot M Repression of Mcl-1 and disruption of the Mcl-1/Bak interaction in myeloma cells couple ER stress to mitochondrial apoptosis. Cancer Lett 2016, 383, 204- 211). The increased ratio of pro-apoptotic proteins leads to initiation of apoptosis that is executed by proteolytically activated caspase 3 . Authors should modify this part. 